\title{
Serum concentrations of some acute phase proteins in cats with anaemia1)
}

\author{
GÜLTEN EMEK TUNA, CEREN DINLER, GAMZE SEVRI EKREN AŞICI*, BÜLENT ULUTAŞ
}

\author{
Department of Internal Medicine, *Department of Biochemistry, \\ Faculty of Veterinary Medicine, Adnan Menderes University, Aydin/Turkey
}

Tuna G. E., Dinler C., Ekren Aşıcı G. S., Ulutaş B.

Serum concentrations of some acute phase proteins in cats with anaemia

\section{Summary}

Serum concentrations of acute phase proteins can provide valuable diagnostic information in the detection and monitoring of disease. The available information on the acute phase response in cats with anaemia is limited. The aim of this study was to retrospectively evaluate serum concentrations of haptoglobin, serum amyloid $A, \alpha 1$ acid glycoprotein and their clinical importance in cats with anaemia. Thirty-four anaemic cats and ten healthy cats were enrolled this study. After individual diagnoses had been established, the cats were divided into three groups (healthy group, haemolytic group and non-haemolytic group). Serum acute phase protein concentrations were analysed using specific commercially available test kits in an ELISA reader device. Serum amyloid $A$ and serum $\alpha 1$ acid glycoprotein concentrations were significantly higher in the anaemic groups compared with the healthy group. Haptoglobin concentrations were significantly higher in cats from the non-haemolytic anaemia group than they were in healthy animals and those from the haemolytic anaemia group. Although serum haptoglobin concentrations were lower than in the healthy group, there was no significant difference between the haemolytic anaemia group and the healthy group. The results of this study suggest that serum amyloid $A$ and $\alpha 1$ acid glycoprotein could be useful in the diagnosis and determination of inflammation in cats with anaemia. Serum haptoglobin depletion may be used for diagnosis of haemolysis in cats with haemolytic anaemia. In addition, this study has contributed to the limited data available on acute phase protein concentrations in cats with anaemia.

Keywords: feline, anaemia, acute phase proteins, serum amyloid A, haptoglobin, alpha-1 acid glycoprotein

Anaemia is defined as reduced haemoglobin $(\mathrm{Hb})$ concentration due to decreased red blood cell (RBC) mass (4). This can be expressed as a packed cell volume (PCV), haematocrit (Hct), RBC count or Hb level below the reference range $(4,5)$. The pathogenesis of anaemia is multifactorial and may be part of a haematological stress syndrome induced by the release of cytokines in response to cellular injury, whether caused by infection, inflammation or malignancy (19). Anaemia is a common haematological alteration in cats, and it is a clinical finding that occurs in association with many diseases. Anaemias can be broadly divided into regenerative (blood loss or haemolytic) and nonregenerative types. Non-regenerative anaemia is associated with chronic inflammatory processes, neoplasia, renal diseases, bone marrow diseases, endocrinopathies such as diabetes mellitus, and occurs as a consequence of some viral diseases (Feline Leukaemia Virus or Feline Immunodeficiency Virus). Regenerative anae-

This study was funded by Adnan Menderes University Research Projects Funding Unit under project number VTF-13014. mia usually develops after blood loss or haemolysis. Haemolysis is frequently associated with many infectious agents, such as Babesia felis, and hemoplasmas (also called hemotropic mycoplasmas, and it was previously known as Haemobartonella felis) (12).

Acute phase proteins (APP) are blood proteins primarily synthesized by hepatocytes as part of the acute phase response (APR), which is triggered by different stimuli, including trauma, infection, stress, neoplasia, and inflammation $(1,16,23)$. APP levels are non-specific variables used in the detection of an inflammatory condition in humans and animals. Serum concentrations of APPs can provide valuable diagnostic information in the detection, prognosis, or monitoring of disease $(1-3,10)$. APPs are classified according to the change in concentration into two groups: positive APPs (e.g. C-reactive protein (CRP), serum amyloid A (SAA), haptoglobin (Hp), and alpha-1 acid glycoprotein (AGP)) if they increase when there is an acute phase response, and negative APPs (e.g. albumin and paraoxonase-1) if they decrease (25). They are further 
classified as major, moderate, or minor, depending on the magnitude and duration of their response to a stimulus. Major proteins represent those that increase 10 to 100 -fold, moderate proteins increase 2- to 10-fold, and minor proteins are characterised by only a slight increase. The magnitude of the increase varies in different species, and each has its major, moderate and minor APPs (1).

In cats, SAA and AGP are recognised as major APPs, whereas $\mathrm{Hp}$ is recognised as a moderate APP (28). Most feline APP studies have been focused on these three APPs. Values of SAA, AGP and Hp are known to be increased in a variety of pathological conditions, such as infection (viral, bacterial), inflammation, injury, renal failure, hospitalization, surgery and neoplasm $(1,25,28)$.

Unfortunately, acute phase proteins in cats with anaemia have not yet been thoroughly investigated. Information available on the acute phase response in cats with anaemia is limited. Ottenjann et al. (19) reported that serum AGP and Hp concentrations were high in cats with anaemia caused by inflammatory diseases, such as abscesses, prothorax and fat necrosis. In a study on feline Hp in cats with hemoplasmas, Harvey and Gaskin (9) report that despite the role of $\mathrm{Hp}$ in binding free haemoglobin after haemolytic crises, Hp concentration did not change in cats with haemolytic anaemia due to hemoplasmas. Korman et al. (13) demonstrated that both Mycoplasma haemofelis (Mhf) and Candidatus Mycoplasma haemominutum (CMhm), despite differing pathogenicity, are associated with an APR in infected cats. SAA was found to have particular promise in this context because it rises higher after Mhf infection than after CMhm infection, and rapidly returns to baseline levels after the acute phase of infection is over. Our study aimed to retrospectively evaluate serum concentrations of SAA, AGP and Hp, as well as their clinical importance in cats with anaemia.

\section{Material and methods}

This study was approved by and performed under the guidelines of the Ethics Committee for Animal Use of University of Adnan Menderes (B.30.2.ADÜ.0.00.00.00/ 050.04/2012/034).

The investigation was carried out on thirty-four anaemic cats $\left(\mathrm{Hct} \leq 27 \%, \mathrm{RBC} \leq 5.5 \times 10^{6} / \mathrm{lL}, \mathrm{Hb} \leq 9 \mathrm{~g} / \mathrm{dL}\right)$ and ten healthy cats. The cats were admitted to the Department of Internal Medicine, Faculty of Veterinary Medicine, the University of Adnan Menderes, for vaccination, clinical examination and therapy. Owner consent to use blood samples for scientific purposes is routinely given at the intake examination. Cats included in the study had not received any medicine before. Basic haematological and biochemical profiles were determined for all cats, which were also given a general clinical examination. Blood samples for haematological and biochemical analyses were obtained by cephalic venipuncture into tubes with and without anti- coagulant. Haematological analyses were performed with an Abacus Junior Vet haematology cell counter (Diatron MI Ltd, Hungary). Blood smears were prepared from all cats, and the slides were examined microscopically. Serum was separated by centrifugation at $3000 \mathrm{~g}$ for 10 minutes. Serum biochemical parameters (alanine aminotransferase, aspartate aminotransferase, alkaline phosphatase, blood urea nitrogen, creatinine, bilirubin and gamma-glutamyl transferase) were analysed with a Samsung Labgeo PT-10 (Samsung Electronics, Seoul, South Korea). The remaining serum was stored at $-20^{\circ} \mathrm{C}$ until analysis for APPs.

Serum AGP (Kamiya Biomedical Company. K-ASSAY, Seattle, USA) concentrations were analysed using specific commercially available test kits in an ELISA reader device. SAA (Solid phase sandwich ELISA kit Tridelta Development LTD. Ireland) and serum Hp (Tridelta Development LTD. Ireland) concentrations were determined using specific commercially available test kits previously validated for use in cats $(10,14)$.

Individual diagnoses were established through detailed clinical examinations and additional laboratory tests and diagnostic procedures (haematology, serum biochemistry, radiography, ultrasonography, cytology ELISA kits and histopathology). Ten cats without clinical abnormalities and with normal routine haematology and biochemical analyses, complemented by negative results for the infectious agents tested, were used as a healthy group. Thirty-four cats with anaemia had different diseases. Ten out of the 34 cats were positive for hemotropic mycoplasmas in blood smears. The 10 cats positive for hemotropic mycoplasmas had bilirubinemia, positive slide agglutination and reticulocytosis. Those 10 cats also had severe or moderate anaemia $(50 \%$ of these animals had severe anaemia, Hct $<15 \%$; and $50 \%$ had moderate anaemia, Hct of 15-19\%). Another 24 cats had non-haemolytic anaemia due to different chronic diseases, such as renal disease, lung disease, liver disease, chronic inflammation, FIV, Feline Infectious Peritonitis (FIP) and neoplasia. After diagnosis, the cats were allocated to the following three groups: healthy group, haemolytic anaemia group and non-haemolytic anaemia group.

Statistical analyses of total leukocyte count (WBC), body temperature (T), SAA, and serum AGP and Hp concentrations were performed using SPSS version 19.0 for Windows (SPSS, Armonk, NY: IBM Corp). The data did not have a normal distribution in a normality test, and the nonparametric Mann-Whitney test was used. $\mathrm{P}<0.05$ was considered statistically significant. 


\section{Results and discussion}

Total leukocyte count, body temperature, SAA, as well as serum AGP and Hp concentrations were compared among all groups. Mean $( \pm \mathrm{SD}) \mathrm{WBC}$, body temperature, SAA, and serum AGP and Hp concentrations are presented in Tab. 1.

The total leukocyte count was significantly higher in cats with non-haemolytic anaemia group compared with healthy and haemolytic anaemia groups (Fig. 1). There were no significant differences in mean body temperature between the groups ( $p>0.05)$ (Fig. 2).

Serum amyloid A and serum AGP concentrations were significantly higher in the anaemic groups compared with the healthy group (Fig. 3 and Fig. 4, respectively). However, SAA and serum AGP concentrations were not significantly different between the haemolytic anaemia group and the non-haemolytic anaemia group.

Haptoglobin concentrations were significantly higher in the non-haemolytic anaemia group compared with the healthy and haemolytic anaemia groups. Although serum Hp concentrations in the healthy group were lower, they were not significantly different from those in the haemolytic anaemia group (Fig. 5).

Recently, acute phase proteins have been of interest in feline medicine as new diagnostic tools. The number of studies in this field continues to increase. The APR is a powerful tool for combating potentially dangerous

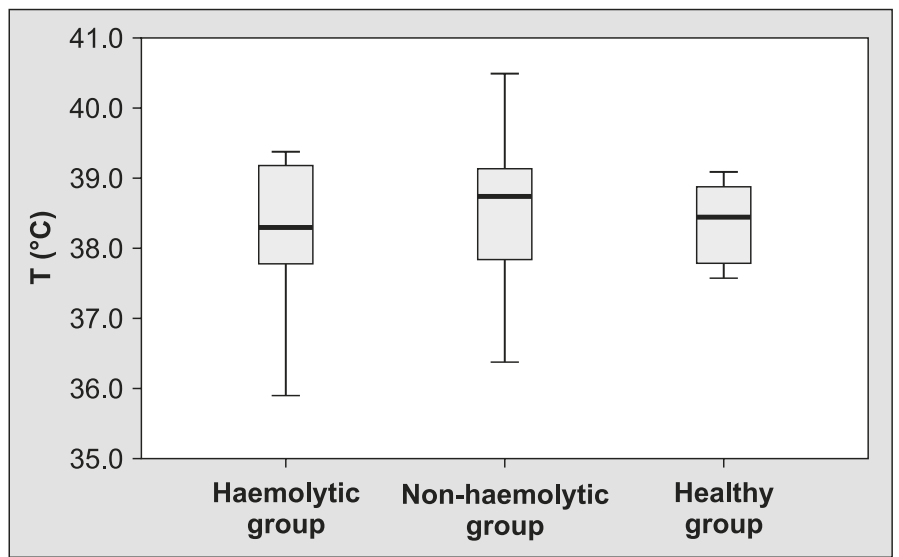

Fig. 2. The mean body temperature for all groups

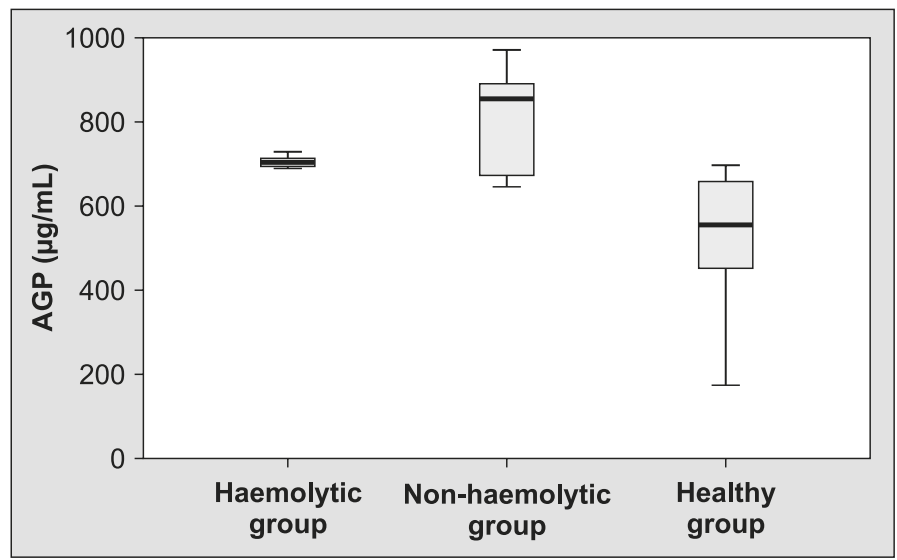

Fig. 4. Serum AGP concentrations for all groups pathogens, which suggests that APPs play a major role in feline inflammation. Diagnostically, the three main hallmarks of the APR (fever, leucocytosis and changes in serum APPs) are all seen in cats with inflammation and can, therefore, be very useful in routine procedures for the early diagnosis of inflammatory conditions, and to monitor progress. APPs are reliable biomarkers and can be used for both diagnostic and research purposes (20).

Specifically, serum amyloid A and AGP are major feline acute phase proteins $(20,24)$. Measurement of SAA and AGP concentrations are considered useful in detecting the presence of inflammation $(17,26,27)$.

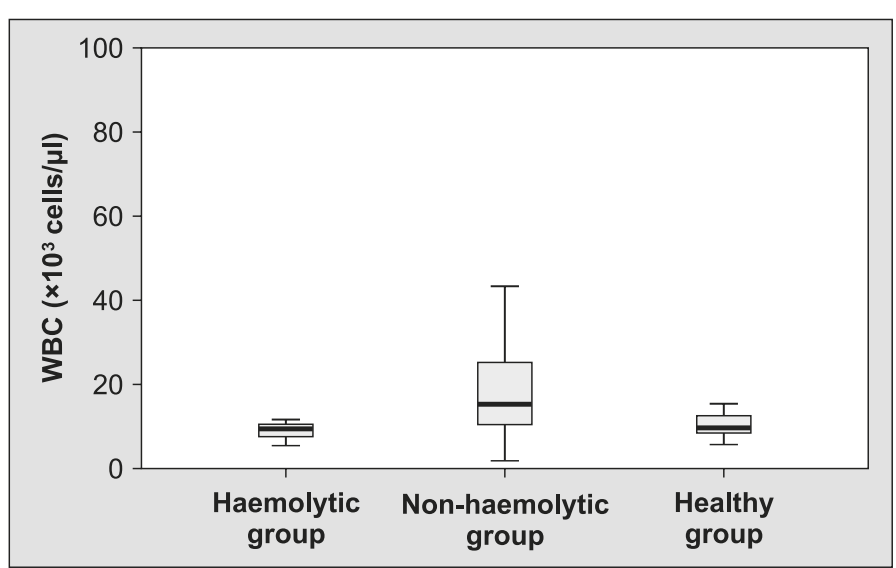

Fig. 1. The mean total leukocyte count for all groups

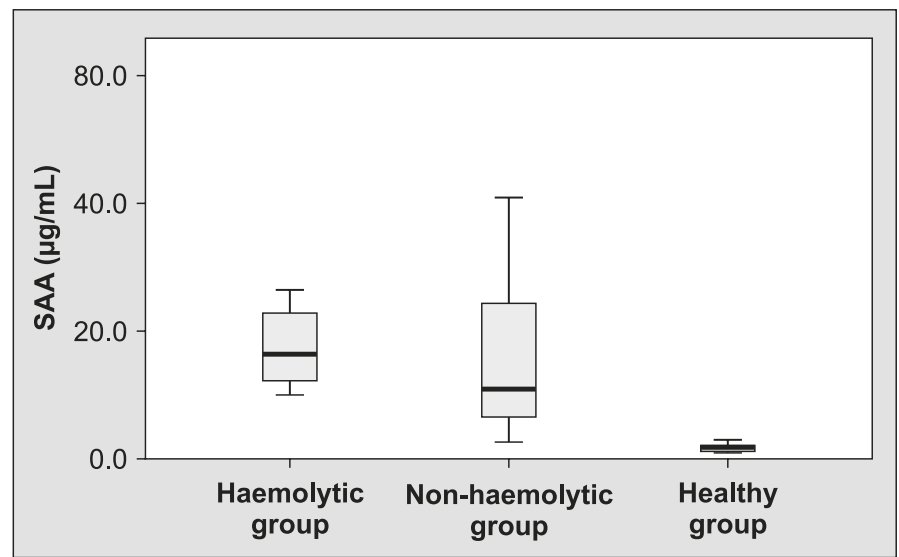

Fig. 3. Serum amyloid A concentrations for all groups

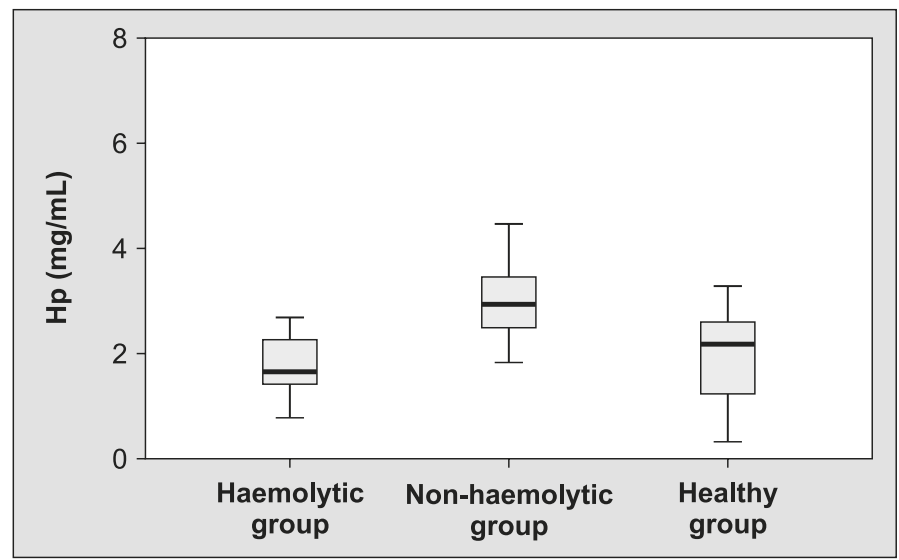

Fig. 5. Serum Hp concentrations for all groups 
In addition, AGP in cat serum or effusion samples is a recognised biomarker of FIP $(6,21,25)$. However, these APP concentrations increase not only in inflammatory diseases, but also in several non-inflammatory disorders, such as neoplasia, diabetes mellitus and hyperthyroidism $(10,22,27)$. In the present study, changes in APPs in cats with anaemia were investigated. The most significant differences in concentrations of SAA and AGP were observed between healthy and anaemic cats. The pathogenesis of anaemia is multifactorial and may be part of a haematological stress syndrome induced by the release of cytokines in response to cellular injury (19). This can explain the reason for the high concentrations of SAA and AGP in cats with anaemia.

This study included 34 cats with anaemia caused by different diseases. Among the 34 cats, ten cats were found positive for hemotropic mycoplasmas. Hemotropic mycoplasmas attach to the surface of feline erythrocytes, causing haemolytic anaemia through the extravascular destruction of erythrocytes by the mononuclear phagocyte system and intravascular lysis by direct damage to the cell membrane, increased osmotic fragility, or both $(11,18)$. In the present study, SAA and AGP concentrations were not significantly different in cats with haemolytic anaemia compared to cats with non-haemolytic anaemia, which points to a possible association of inflammation with the presence of anaemia in cats. SAA and AGP concentrations were significantly higher in both anaemic groups compared to the control group, which confirms the role of SAA and AGP as markers of systemic inflammation in anaemia.

Apart from sporadic studies in which Hp was included among other indicators of inflammation in specific diseases (7), the first report on feline $\mathrm{Hp}$ was by Harvey and Gaskin (9), who described Hp increases in cats with several experimentally induced pathological conditions. In cats, $\mathrm{Hp}$ is considered to be a moderate APP, as its levels may be 2-10 times greater during inflammation (15). Hp concentration is increased in inflammatory diseases in cats, which is indicative of inflammatory processes (15). In contrast to other APPs, serum Hp concentrations were significantly higher in the non-haemolytic group compared to those in the haemolytic and healthy groups. In this context, it is important to note that $\mathrm{Hp}$ binds to free haemoglobin, and the complex is cleared by the reticuloendothelial system, resulting in a decrease in serum Hp concentrations (29). Normal levels of Hp in cats with haemolytic anaemia might be the result of an imbalance between the synthesis and the clearance of this protein from the circulation. Similar to our results, Harvey and Gaskin (9) report that Hp did not change in cats with haemolytic anaemia due to hemoplasmas. In contrast, a marked decrease in serum Hp concentration was found in different species with haemolysis $(8$,
29). It may be related to the magnitude of haemolysis. Findings on Hp concentration in the non-haemolytic group are similar to those by Ottenjann et al. (19), who reported increased $\mathrm{Hp}$ in anaemic cats with inflammatory conditions.

In conclusion, the results of the present study suggest that APPs, including SAA and AGP, have a higher diagnostic sensitivity than leukocytosis and fever in the diagnosis of an inflammatory condition in cats with anaemia, and that serum Hp depletion may used for diagnosis of haemolysis in cats with haemolytic anaemia. Although the duration and severity of anaemia were not determined in the present study, the results obtained will be useful for clinicians and scientific researchers. This study has supplemented the limited data available on acute phase protein concentrations in cats with anaemia.

\section{References}

1. Ceron J. J., Eckersall P. D., Martýnez-Subiela S.: Acute phase proteins in dogs and cats: current knowledge and future perspectives. Vet. Clin. Pathol. 2005, 34, 85-99.

2. Dabrowski R., Kostro K., Lisiecka U., Szczubial M., Krakowski L.: Usefulness of C-reactive protein, serum amyloid A component, and haptoglobin determinations in bitches with pyometra for monitoring early post-ovariohysterectomy complications. Theriogenology 2009, 72, 471-479.

3. Eckersall P. D., Bell R.: Acute phase proteins: biomarkers of infection and inflammation in veterinary medicine. Vet. J. 2010, 85, 23-27.

4. Fleischman W.: Anemia: determining the cause. Compend. Contin. Educ. Vet. 2012, 34, 1.

5. Furman E., Leidinger E., Hooijberg E. H., Bauer N., Beddies G., Moritz A.: A retrospective study of 1,098 blood samples with anemia from adult cats: frequency, classification, and association with serum creatinine concentration. J. Vet. Intern. Med. 2014, 28, 1391-1397.

6. Giordano A., Spagnolo V., Colombo A., Paltrinieri S.: Changes in some acute phase protein and immunoglobulin concentrations in cats affected by feline infectious peritonitis or exposed to feline coronavirus infection. Vet. J. 2004, 167, 38-44.

7. Gouffaux M., Pastoret P. P., Henroteaux M., Massip A.: Feline infectious peritonitis proteins of plasma and ascitic fluid. Vet. Pathol. 1975, 12, 335-348.

8. Gruys E., Toussaint M. J. M., Niewold T. A., Koopmans S. J.: Acute phase reaction and acute phase proteins. J. Zhejiang Univ. Sci. B 2005, 6, 1045-1056.

9. Harvey J. W., Gaskin J. M.: Experimental feline haemobartonellosis. J. Am. Anim. Hosp. Assoc. 1977, 13, 28-38.

10. Kann R. K., Seddon J. M., Henning J., Meers J.: Acute phase proteins in healthy and sick cats. Res. Vet. Sci. 2012, 93, 649-654.

11. Kewish K. E., Appleyard G. D., Myers S. L., Kidney B. A., Jackson M. L.: Mycoplasma haemofelis and Mycoplasma haemominutum detection by polymerase chain reaction in cats from Saskatchewan and Alberta. Can. Vet. J. 2004, 45, 749-752.

12. Kohn B., Weingart C., Eckmann V., Ottenjann M., Leibold W.: Primary ImmuneMediated Hemolytic Anemia in 19 Cats: Diagnosis, Therapy, and Outcome (1998-2004). J. Vet. Intern. Med. 2006, 20, 159-166.

13. Korman R. M., Cerón J. J., Knowles T. G., Barker E. N., Eckersall P. D., Tasker $S$.: Acute phase response to Mycoplasma haemofelis and 'Candidatus Mycoplasma haemominutum' infection in FIV-infected and non-FIV-infected cats. Vet. J. 2012, 93, 433-438.

14. Leal R. O., Gil S., Sepúlveda N., McGahie D., Duarte A., Niza M. M. R. E., Tavares $L$ : : Monitoring acute phase proteins in retrovirus infected cats undergoing feline interferon- $\omega$ therapy. J. Small Anim. Pract. 2014, 55, 39-45.

15. Mattsson J.: Evaluation of in vitro diagnostic (point-of-care) system for quantification of the acute phase protein haptoglobin in cats. Master Thesis, Master of Science in Biomedical Laboratory Science, Göteborg, Sweden 2014.

16. Murata H., Shimada N., Yoshioka M.: Current research on acute phase proteins in veterinary diagnosis: an overview. Vet. J. 2004, 168, 2840.

17. Nakayama T., Sonoda S., Urano T., Yamada T., Okada M.: Monitoring both serum amyloid protein A and C-reactive protein as inflammatory markers in infectious diseases. Clin. Chem. 1993, 39, 293-297. 
18. Nibblett B. M. D., Snead E. C., Waldner C., Taylor S. M., Jackson M. L., Knorr L. M.: Anemia in cats with hemotropic mycoplasma infection: Retrospective evaluation of 23 cases (1996-2005). Can. Vet. J. 2009, 50, 1181-1185.

19. Ottenjann M., Weingart C., Arndt G., Kohn B.: Characterization of the anemia of inflammatory disease in cats with abscesses, pyothorax or fat necrosis. J. Vet. Intern. Med. 2006, 20, 1143-1150.

20. Paltrinieri S.: The feline acute phase reaction. Vet. J. 2008, 177, 26-35.

21. Paltrinieri S., Marchini I., Gelain M. E.: Flow cytometric detection of alpha-1-acid glycoprotein on feline circulating leucocytes. Aust. Vet. J. 2012, 90, 291-296

22. Paltrinieri S., Metzger C., Battilani M., Pocacqua V., Gelain M. E., Giordano A.: Serum a1-acid glycoprotein (AGP) concentration in non-symptomatic cats with feline coronavirus (FCoV) infection. JFMS 2007b, 9, 271-277.

23. Petersen H. H., Nielsen J. P., Heegaard P. M.: Application of acute phase protein measurements in veterinary clinical chemistry. Vet. Res. 2004, 35, 163-187

24. Sasaki K., Ma Z., Khatlani T. S., Okuda M., Inokuma H., Onishi T.: Evaluation of feline serum amyloid A (SAA) as an inflammatory marker. JVMS 2003 $65,545-548$
25. Schmidt E. M., Eckersall P. D.: Acute phase proteins as markers of infectious diseases in small animals. Acta Vet. Beograd 2015, 65, 149-161.

26. Tamamoto T., Ohno K., Ohmi A., Goto-Koshino Y., Tsujimoto H.: Verification of measurement of the feline serum amyloid A(SAA) concentration by human SAA turbidimetric immunoassay and its clinical application. J. Vet. Med. Sci. 2008, 70, 1247-1252.

27. Tamamoto T., Ohno K., Takahashi M., Nakashima K., Fujino Y., Tsujimoto H.: Serum amyloid A as a prognostic marker in cats with various diseases. J. Vet. Diagn. Invest. 2013, 5, 428-432.

28. Tuna G. E., Ulutaş B.: Acute phase proteins as biomarkers of disease. J. Vet. Sci. Intern. Med. Special Topics 2015, 1, 8-19.

29. Ulutas P. A., Ulutas B., Sarierler M., Bayramll G.: Serum haptoglobin and ceruloplasmin concentrations in dogs with various diseases. The Journal of the Faculty of Veterinary Medicine Istanbul University 2007, 33, 35-42.

Corresponding author: Gülten Emek TUNA, DVM, PhD; Department of Internal Medicine, Faculty of Veterinary Medicine, Adnan Menderes University, Isikli, Aydın, 09017, Turkey; e-mail: emektuna@adu.edu.tr 